\title{
FENTANYL: EFECTOS ADVERSOS EN ANESTESIA SUBARACNOIDEA CON BUPIVACAÍNA
}

Luis Eduardo Reyes MD*, Manuel Robayo MD**, Camilo Andrés Preciado Aponte MD***, Javier Alfonso Pulido MD ${ }^{* * * *}$, Julián Andrés Navarro Castro MD ${ }^{* * * * *}$, Gabriel Ríos Gamboa MD ${ }^{* * *}$

\section{Resumen}

Los efectos adversos de los opioides son el resultado de interacciones con sus receptores a nivel cerebral. Cuando se administran por vía intratecal se ha descrito depresión respiratoria, aunque con menor frecuencia que en uso endovenoso. Objetivo: describir la ocurrencia de efectos adversos en pacientes llevados a cirugía en el Hospital de San José de Bogotá D.C. que recibieron fentanyl intratecal como adición a bupivacaína hiperbárica. Materiales y métodos: estudio descriptivo de una cohorte de pacientes llevados a cirugía en el Hospital de San José entre $1^{\circ}$ de octubre de 2007 y 30 de septiembre de 2009, que recibieron anestesia subaracnoidea aplicando fentanyl intratecal y bupivacaína hiperbárica. Se incluyeron los pacientes de 18 a 65 años, las no gestantes y aquellos sin conversión a anestesia general. Resultados: se estudiaron 313 pacientes, 39,9\% mujeres con edad promedio de 42 años (DE:12,7), clasificación ASA distribuida en ASA I, 60,7\%; ASA II, 33,3\%; ASA III, 5,7\% y ASA IV, 0,3\%. Los efectos adversos más comunes fueron náuseas $8,6 \%$ (n:27), prurito 6,7\%(n:21), vómito 2,2\% (n:7) y bradicardia 2,2\% (n:7). La depresión respiratoria se presentó en 1,3\% (n:4). Conclusiones: la frecuencia de depresión respiratoria que reportamos se encuentra en el rango de la literatura; sin embargo, hay que considerar que no existe consenso en la manera como se mide. Los demás eventos adversos fueron menos que los reportados.

Palabras clave: efectos adversos, fentanyl, intratecal, depresión respiratoria, apnea, prurito, náuseas, vómito, anestesia subaracnoidea.

Abreviaturas: FEN, fentanyl; BUP, bupivacaína; DR, depresión respiratoria; EA, efecto (s) adverso (s).

\section{ADVERSE SIDE EFFECTS OF FENTANYL PLUS BUPIVACAINE SUBARACHNOID ANESTHESIA}

\section{Abstract}

Adverse effects of opioids result of their interactions with opioid brain receptors. Intrathecal administration of fentanyl may induce respiratory depression but less frequently than when intravenously administered. Objective: to describe the frequency of adverse side effects in surgical patients at Hospital de San José who received intrathecal fentanyl plus hyperbaric bupivacaine. Materials and Methods: descriptive study of a cohort of patients who underwent surgery at Hospital de San José between October 12007 and September 30 2009, who received intratecal fentanyl plus hyperbaric bupivacaine. Patients aged 18 to 65 years, nonpregnant women and those who were not converted

Fecha recibido: marzo 8 de 2010 Fecha aceptado: junio 23 de 2010

* Anestesiólogo. Jefe del Servicio de Anestesiología, Hospital de San José, Bogotá D.C. Colombia.

** Anestesiólogo, Servicio de Anestesia, Hospital de San José y Hospital El Carmen, Bogotá D.C. Colombia.
*** Residente III de Anestesiología y Reanimación. Fundación Universitaria de Ciencias de la Salud. Bogotá D.C. Colombia.

**** Anestesiólogo Servicio de Anestesiología, Hospital de San José y Clínica de Occidente, Bogotá D.C. Colombia.

*****Anestesiólogo, Servicio de Anestesiología, Hospital de San José, Hospital Universitario La Samaritana, Bogotá D.C. Colombia. 
into general anesthesia were included. Results: 313 patients were studied, $39.9 \%$ women with mean age 42 years (SD: 12.7), classified as: ASA I 60.7\%; ASA II, 33.3\%; ASA III, 5.7\% and ASA IV, 0.3\%. The most common adverse side effects were, nausea $8.6 \%$ (n: 27), pruritus $6.7 \%$ (n: 21), vomiting $2.2 \%$ (n: 7) and bradycardia $2.2 \%$ (n: 7 ). Respiratory depression presented in $1.3 \%$ ( $\mathrm{n}: 4)$. Conclusions: our report of the frequency of respiratory depression is within that described in literature; however, it must be considered that the measuring methods were not consistent. Other adverse events were lower than those reported.

Key words: adverse side effects, fentanyl, intrathecal, respiratory depression, apnea, pruritus, nausea, vomiting, subarachnoid anesthesia.

\section{Introducción}

La administración subaracnoidea de anestésicos locales y opioides, es una excelente técnica anestésica y de control del dolor después de procedimientos en miembros inferiores y cirugías torácicas, abdominales o pélvicas. Sin embargo, se describen EA con el uso de opioides intratecales de los cuales los más comunes son prurito $(30 \%)^{1}$ náuseas y vómito $(25 \%),{ }^{1,2}$ retención urinaria $(10 \%-53 \%)^{3,4}$ y el más temido la DR $(3 \%){ }^{2}$ todo esto como resultado de interacciones con los receptores opioides a nivel cerebral. ${ }^{5}$

El manejo de estos eventos puede requerir desde intervenciones menores como generar un estímulo verbal para logar respuesta en casos de DR leve, hasta la necesidad de iniciar ventilación mecánica en los casos severos. Además de intervenciones para el manejo de otras situaciones como sonda vesical cuando hay retención urinaria o el manejo del vómito posoperatorio, que conducen al aumento de la morbilidad, estancia hospitalaria y costos. ${ }^{6}$

El objetivo de este estudio es identificar la frecuencia de EA con el uso de FEN intratecal asociado con BUP, considerando que de las 12.000 cirugías al año que se realizan en el Hospital de San José, el 35\% son bajo anestesia regional, de las cuales $85 \%$ corresponden a la técnica subaracnoidea o espinal. No conocemos la frecuencia de EA en la población llevada a cirugía con esta técnica anestésica en la población de nuestro hospital.

\section{Materiales y métodos}

Con aprobación del comité de investigaciones y ética de la Facultad de Medicina de la Fundación Universitaria de Ciencias de la Salud y el Hospital de San José, se dio inicio a un estudio descriptivo de cohorte prospectiva en el que ingresaron pacientes llevados a cirugía, que recibieron vía subaracnoidea FEN intratecal asociado con BUP hiperbárica al 0,5\% como una opción establecida en el plan anestésico, en el período comprendido entre $1^{\circ}$ de octubre 2007 y 30 de septiembre 2009. Ingresaron quienes tenían entre 18 y 65 años y se excluyeron las pacientes obstétricas, aquellos con conversión a anestesia general durante el procedimiento y cuando se usaron opioides seis horas antes de la cirugía. Todos firmaron el consentimiento informado.

Se describen las variables edad, sexo, ASA de acuerdo con la clasificación del estado físico (American Society of Anesthesiologists), ${ }^{7}$ tabaquismo definido como consumo de al menos un cigarrillo en el último mes según el ATP III ${ }^{8}$ prioridad quirúrgica (programada o de urgencia), tipo de procedimiento quirúrgico diferenciando entre abdominal, pélvico y de miembros inferiores, dosis de anestésico local, dosis de FEN subaracnoideo y nivel anestésico dado por el bloqueo sensitivo alcanzado.

Los EA registrados fueron DR cuando se identificó una desaturación significativa ( $4 \%$ o más) de la saturación arterial de oxígeno ${ }^{9}$ a partir de la basal previa a la inducción anestésica medida con oximetría de pulso; bradicardia (frecuencia cardíaca menor o igual a 60 latidos por minuto ${ }^{10}$ independiente de la necesidad de intervención farmacológica, prurito (según autorreporte dado por el paciente) y retención urinaria reportada como el requerimiento de sonda vesical sin indicación relacionada con el procedimiento quirúrgico.

Dado que el uso simultáneo de benzodiacepinas con opiodes aumenta el riesgo de $\mathrm{DR}^{5}$, se determinó el uso de hipnóticos como la necesidad de cualquier dosis de midazolam endovenoso tanto en salas como en la uni- 
dad de cuidado posanestésico. La aparición de EA se documentó tanto en salas por parte del anestesiólogo, como en la unidad de cuidados posanestésicos por el personal de enfermería entrenado para identificarlos y registrarlos en el formato de recolección de datos. Todos fueron observados desde el inicio del evento anestésico hasta su egreso de la unidad de cuidado posanestésico. El análisis de resultados se realizó en el programa STATA $10 \AA$. La estadística descriptiva se usó para presentar los datos.

\section{Resultados}

A través del formato de recolección se registraron datos de 320 pacientes desde octubre 1 de 2007 hasta septiembre 30 de 2009. Se excluyeron siete casos por inadecuada recolección de información. Se evaluaron 313: $60,1 \%$ (188) hombres, promedio de edad de 42 años (DE 12.7); según la clasificación del estado físico del ASA, el mayor número de pacientes correspondió a la categoría
ASA I con un total de $60,7 \%$ (190), seguido por ASA II $33,3 \%$ (104). Los procedimientos realizados como urgencia corresponden al 9,6\% (30). Las demás características de la población se presentan en la Tabla 1.

El EA más frecuente fue náuseas 8,6\% (27), seguido por prurito $6,7 \%$ (21), vómito $2,2 \%$ (7) y bradicardia $2,2 \%$ (7). La frecuencia de DR fue de 1,3\% (4) (Tabla 2). Según el nivel anestésico obtenido, se consideró como bloqueo sensitivo alto T5 la ausencia de sensación a la presión con los dedos del evaluador. De los que alcanzaron dicho nivel, tres manifestaron náuseas, uno bradicardia y ninguno vómito ni desaturación significativa. Las dosis promedio de FEN administrado por vía intratecal fue de 20.6 (DE 4.08), sin encontrar cuándo hubo o no efectos adversos. Al analizar los casos con DR se encontraron tres que tenían clasificación ASA I, dos eran fumadores, tres tuvieron procedimientos quirúrgicos de los miembros inferiores y dos habían recibido hipnóticos en forma concomitante (Tabla 3).

\begin{tabular}{|c|c|c|c|c|c|c|}
\hline \multirow[t]{2}{*}{ Sexo } & \multicolumn{2}{|c|}{ Hombres } & \multicolumn{2}{|c|}{ Mujeres } & \multicolumn{2}{|c|}{ Total } \\
\hline & $\begin{array}{r}N \\
188\end{array}$ & $\begin{array}{l}\% \\
(60,1)\end{array}$ & $\begin{array}{r}n \\
125\end{array}$ & $\begin{array}{l}\% \\
(39,9)\end{array}$ & $\begin{array}{r}n \\
313\end{array}$ & $\%$ \\
\hline $\begin{array}{l}\text { Edad, años promedio (DE*) } \\
\text { ASA } †\end{array}$ & 40.4 & $(13,6)$ & 44.3 & $(10,9)$ & 42 & $(12,7)$ \\
\hline 1 & 123 & $(65,5)$ & 67 & $(53,6)$ & 190 & $(60,7)$ \\
\hline II & 55 & $(29,2)$ & 49 & $(39,2)$ & 104 & $(33,3)$ \\
\hline III & 9 & $(4,8)$ & 9 & $(7,2)$ & 18 & $(5,7)$ \\
\hline IV & 1 & $(0,5)$ & 0 & 0 & I & $(0,3)$ \\
\hline \multicolumn{7}{|l|}{ Fumador } \\
\hline no & 122 & $(64,9)$ & 103 & $(82,4)$ & 225 & $(7 I, 9)$ \\
\hline fumador $\ddagger$ & 43 & $(22,9)$ & 8 & $(6,4)$ & 51 & $(16,3)$ \\
\hline exfumador & 23 & $(12,2)$ & 14 & $(11,2)$ & 37 & $(11,8)$ \\
\hline \multicolumn{7}{|l|}{ Prioridad } \\
\hline programada & 166 & $(88,3)$ & 117 & $(93,6)$ & 283 & $(90,4)$ \\
\hline urgencia & 22 & $(11,7)$ & 8 & $(6,4)$ & 30 & $(9,6)$ \\
\hline \multicolumn{7}{|l|}{ Tipo de procedimiento } \\
\hline miembros inferiores & 140 & $(74,5)$ & 69 & $(55,2)$ & 209 & $(66,7)$ \\
\hline pélvico & 25 & $(13,3)$ & 52 & $(4 I, 6)$ & 77 & $(24,6)$ \\
\hline abdominal & 23 & $(12,2)$ & 4 & $(3,2)$ & 27 & $(8,7)$ \\
\hline Dosis de BUP mg, promedio (DE) & 15 & $(3,07)$ & 14 & $(3,04)$ & 14.6 & $(3,09)$ \\
\hline mínima - máxima & 5 & 20 & 5 & 20 & 5 & 20 \\
\hline Dosis de FEN $\mu \mathrm{g}$, promedio (DE) & 20.5 & $(3,6)$ & 20.9 & $(4,6)$ & 20.6 & $(4,08)$ \\
\hline mínima - máxima & 10 & 50 & 10 & 50 & & $10 \quad 50$ \\
\hline Tiempo anestésico, mediana (RIQ) & 90 & $(60-120)$ & 90 & $(60-120)$ & 90 & $(60-120)$ \\
\hline
\end{tabular}

* Desviación estándar; † clasificación de la Sociedad Americana de Anestesiología; $\ddagger$ cualquier cigarrillo en el último mes (ATP III); § rango intercuartílico. 


\begin{tabular}{|c|c|c|c|c|c|c|}
\hline & \multicolumn{2}{|c|}{ Salas } & \multicolumn{2}{|c|}{ Recuperación } & \multicolumn{2}{|c|}{ Total \% } \\
\hline & & & $\mathbf{n}$ & $\%$ & & \\
\hline Desaturación significativa* & 4 & $(1,3)$ & 0 & 0 & 4 & $(1,3)$ \\
\hline Bradicardia $†$ & 4 & $(1,3)$ & 3 & $(0,96)$ & 7 & $(2,2)$ \\
\hline Con requerimiento de intervención $\ddagger$ & 0 & 0 & 3 & $(0,96)$ & 3 & $(0,96)$ \\
\hline Prurito & 20 & $(6,4)$ & 1 & $(0,3)$ & 21 & $(6,7)$ \\
\hline Náuseas§ & 20 & $(6,4)$ & 8 & $(2,6)$ & 27 & $(8,6) \S$ \\
\hline Vómito & 5 & $(1,6)$ & 2 & $(0,6)$ & 7 & $(2,2)$ \\
\hline Retención urinaria Ø & $\mathrm{nv}$ & $\mathrm{nv}$ & 5 & $(1,6)$ & 5 & $(1,6)$ \\
\hline
\end{tabular}

Se describe frecuencia de eventos adversos en salas y en unidad de recuperación posanestésica.* Desaturación significativa: disminución de más del $4 \%$ del valor basal de la $\mathrm{SaO}_{2}$ medida por oximetría de pulso; $\mathrm{t}$ todos los eventos con frecuencia cardíaca menor de 60 latidos por minuto, independiente de necesidad de intervención; ningún paciente presentó asociación con bradicardia y desaturación significativa; $\ddagger$ requerimiento de atropina; $\S$ un paciente presentó evento en salas y en recuperación, nv: no valorable; TI ningún evento de retención urinaria requirió manejo con sonda vesical.

\section{Discusión}

En la práctica clínica es común la adición de FEN a la anestesia subaracnoidea por los efectos benéficos que tiene en la analgesia posoperatoria. Sin embargo, la lite-

\begin{tabular}{|c|c|c|c|c|}
\hline \multirow[b]{2}{*}{ Edad. años promedio (DE*) } & \multicolumn{2}{|c|}{$\begin{array}{c}\text { Sí } \\
n=4\end{array}$} & \multicolumn{2}{|c|}{$\begin{array}{c}\text { No } \\
n=309\end{array}$} \\
\hline & 44.5 & $(15,6)$ & 41.9 & $(12,7)$ \\
\hline mín-máx & 23 & 58 & 18 & 77 \\
\hline \multicolumn{5}{|l|}{ ASA† (\%) } \\
\hline 1 & 3 & (75) & 187 & $(60,5)$ \\
\hline II & 1 & $(25)$ & 103 & $(33,3)$ \\
\hline III & 0 & & 18 & $(5,8)$ \\
\hline IV & 0 & & 1 & $(0,3)$ \\
\hline \multicolumn{5}{|l|}{ Fumador (\%) } \\
\hline no & 2 & (50) & 223 & $(72,1)$ \\
\hline fumador $\ddagger$ & I & $(25)$ & 50 & $(16,2)$ \\
\hline exfumador & 1 & $(25)$ & 36 & $(11,7)$ \\
\hline \multicolumn{5}{|l|}{ Prioridad (\%) } \\
\hline urgencia & 0 & & 30 & $(9,8)$ \\
\hline programada & 4 & $(100)$ & 279 & $(90,2)$ \\
\hline \multicolumn{5}{|l|}{ Tipo de procedimiento (\%) } \\
\hline abdominal & I & $(25)$ & 26 & $(8,4)$ \\
\hline pélvico & 0 & & 77 & $(25)$ \\
\hline miembros inferiores & 3 & (75) & 206 & $(66,6)$ \\
\hline Nivel anestésico alto§ (\%) & $\mathbf{0}$ & & 26 & $(8,4)$ \\
\hline $\begin{array}{l}\text { Uso de hipnóticos (\%) } \\
\text { §T5 o superior. }\end{array}$ & 2 & (50) & 59 & $(19)$ \\
\hline
\end{tabular}

ratura reporta discrepancias tanto en la frecuencia de estos efectos como en las definiciones de los desenlaces clínicos de interés, en particular la DR. Esta la encontramos en el 1,3\%, definida como desaturación significativa. Otros estudios ${ }^{1,4,11-13}$ no reportan este evento, pero sin hacer una clara descripción de la manera como se midió. En contraste, el estudio de Gwirtz ${ }^{2}$ reportó $3 \%$ en un estudio de siete años de seguimiento de 5.969 pacientes quirúrgicos, definiéndola como niveles de $\mathrm{CO} 2>50$ $\mathrm{mm} \mathrm{Hg}$ o frecuencia menor de ocho respiraciones por minuto. Los resultados entre estudios no son estrictamente similares, teniendo en cuenta que los procedimientos y las poblaciones también difieren entre ellos.

Además, es necesario aclarar que la saturación de oxígeno inicial medida por pulsoximetría continua no puede ser un valor absoluto ya establecido, pues existe una amplia variabilidad. Un valor que para algunos pacientes puede llegar a ser anormal bajo, para otros sería el esperado de acuerdo con su condición clínica previa. Por tal razón, más que la disminución del valor por debajo de un número fijo, consideramos significativa una reducción de la saturación de oxígeno en un $4 \%$ o más del valor inicial registrado. La distribución por sexos de la DR fue una mujer $(0,8 \%)$ por tres hombres $(1,6 \%)$, característica no descrita en los estudios revisados. En cuanto al momento en que se presentó el evento, la DR siempre ocurrió en el transoperatorio y no fue necesario el manejo invasivo de la vía aérea ni requirió reversión del efecto con naloxona. 
En el estudio de Reuben S. S. y col. ${ }^{11}$ el uso de FEN intratecal en sesenta casos de cirugía para revascularización de miembros inferiores, con dosis de 40 a $50 \mathrm{mg}$ por vía intratecal, se logró una analgesia expresada como excelente sin presencia de eventos de DR, hipoxemia ni alteraciones hemodinámicas. El prurito apareció con dosis mayores de $50 \mathrm{mg}$. En el estudio de Kristiina S. Kuusniemi y col. ${ }^{1}$ con la adición de $25 \mathrm{mg}$ de FEN a BUP para determinar el aumento del bloqueo sensitivo y motor en cirugía urológica, el prurito fue el EA más común (22,5\%), seguido de escalofrío (10\%), vómito $(1,3 \%)$ y náuseas $(1,3 \%)$, pero sin ningún caso de DR. Se reportó bradicardia $(3,8 \%)$ que incluso requirió algún tipo de manejo para su corrección. De igual manera en otro estudio realizado por J.A Vaughan y col. ${ }^{12}$ al comparar el uso de FEN y diamorfina en una técnica de anestesia intratecalintradural para analgesia en 62 mujeres embarazadas, el prurito fue el EA más común presentándose en cuatro de 31 cuando administró FEN y en una de 28 con el uso de diamorfina, pero no hubo náuseas, vómito ni depresión respiratoria. Otras series describen resultados similares con respecto a la presencia de EA siendo la DR muy poco frecuente o incluso ausente. ${ }^{14-20}$

El diseño de nuestro estudio plantea hacer descripción de frecuencias, no buscar asociaciones. Tampoco se midieron variables concernientes a los beneficios y/o efectos terapéuticos, los cuales ya están descritos en la literatura. Hay que tener en cuenta la proporción de prioridades de los procedimientos manejados con anestesia subaracnoidea en nuestra institución, ya que apenas el 9,6\% fueron urgencias, lo que no nos permite analizar con precisión la relación de la prioridad del procedimiento con la presencia de EA. La mayoría en el estudio se sometieron a cirugía de miembros inferiores, lo cual influye para que los EA fueran más frecuentes en este grupo de procedimientos.

Existen trabajos que describen efectos adversos con el uso de FEN intratecal en procedimientos que incluyen diferentes sitios anatómicos. Sudarshan y col. ${ }^{21}$ lo emplearon en treinta casos de toracotomia buscando determinar la eficacia analgésica de dosis intermitentes a través de catéteres microespinales en el espacio subaracnoideo lumbar, demostrando un rápido inicio de la analgesia, bajos niveles de dolor en reposo y con la tos, sin DR ya fuese temprana o tardía, a pesar del sitio anatómico del procedimiento quirúrgico. Techanivate y col. ${ }^{4}$ midieron la eficacia en apendicectomías de la mezcla BUP al 5\% hiperbárica más FEN, comparada con BUP hiperbárica al 5\% más solución salina, encontrando no sólo prolongación de la analgesia de la mezcla con FEN como efecto terapéutico, sino que además no existieron diferencias significativas en la incidencia de náusea, vómito, hipotensión y retención urinaria, sumado al hecho de que ningún paciente presentó DR.

Herpolsheimer y col. ${ }^{3}$ usaron mezcla de FEN $25 \mathrm{mg}$ y morfina $0.25 \mathrm{mg}$ en una inyección intratecal lumbar única para analgesia obstétrica en trabajo de parto, reportando EA que incluían prurito (81,3\%), retención urinaria $(53,3 \%)$, náuseas y vómito (44\%). Se presentaron depresión respiratoria $(1,3 \%)$ y sobresedación $(1,3 \%) \sin$ desaturación por debajo de $90 \%$ y respondieron con el solo llamado. De esta manera, se puede afirmar que independiente del lugar anatómico donde se realizó el procedimiento quirúrgico, la frecuencia de EA fue similar. Lo mismo puede observarse cuando se considera el estado físico del ASA, ya que la mayoría de EA se presentaron con ASA I, pero a su vez fue el grupo con mayor número de participantes. En otras series no se evidenció relación entre el estado físico del ASA y los efectos secundarios relacionados con el uso de FEN intratecal.

Pudo observarse que la dosis promedio de FEN en quienes hubo algún EA no tuvo una diferencia importante con respecto a los que no los presentaron. Reuben y col. ${ }^{11}$ evaluaron las dosis y las respuestas con FEN intratecal en una población de pacientes con revascularización de miembros inferiores, usando dosis de $0,5,10,20,40$ y $50 \mathrm{mg}$ de FEN posoperatorio a través de un catéter espinal. Si bien se apreció una analgesia satisfactoria a partir de los $20 \mathrm{mg}$ no existieron EA en los diferentes grupos, exceptuando con el uso de $50 \mathrm{mg}$ en donde $50 \%$ presentó prurito. Esto nos indica que la probabilidad de EA viene ligada a la dosis administrada y que en nuestro estudio no se puede describir tal hallazgo debido a que en la mayoría la dosis fue similar.

Con respecto al nivel sensitivo, los EA se encontraron por lo regular por debajo de T5, lo que está en relación 
con la proporción de pacientes en este grupo que fue de $91,69 \%$. Algunos autores citan la probabilidad de que independiente del área de punción para colocar el fármaco intratecal, puede haber una amplia distribución del medicamento por las características intrínsecas de los opioides conllevando en especial a DR. ${ }^{22}$

Hamber y col. ${ }^{23}$ en una revisión de la literatura realizada en 1999 y que recogía estudios a partir de 1980, describieron que según el perfil lipofílico o hidrofílico de un opioide se determinaba la distribución del fármaco en el líquido cefalorraquídeo y a su vez la posibilidad de EA. La DR se presentó con mayor frecuencia con el uso de morfina intratecal, ya que su característica hidrofílica le permitió una eliminación más lenta, con una importante prolongación de la analgesia de hasta 24 horas en algunos casos. Dada su alta permanencia, se asociaba con DR tardía, casi siempre después de ocho a diez horas de administrado el medicamento. Otros opioides lipofílicos como el FEN se asoció con menor frecuencia de DR ya que su eliminación en líquido cefalorraquídeo es más rápida, con una duración de la analgesia más corta, entre una y cuatro horas, y la posibilidad de presentar un pico de DR entre cinco y veinte minutos de administrado el medicamento, asociado con la dosis usada. Bernards ${ }^{24}$ en revisiones más recientes en 2002 y 2004, anotó hallazgos similares.

Este mismo hecho fue demostrado por Ummenhofer y col..$^{22}$ en un modelo animal usando cerdos, al administrar morfina, FEN alfentanyl y sufentanyl intratecales a nivel de L3, para analizar después el líquido cefalorraquídeo por microdiálisis a nivel de L2, T11, T7, T3 y del espacio epidural en L2 cada diez a quince minutos. El modelo demostró que el alfentanyl y el FEN se eliminan más rápido que la morfina y el sufentanyl. Estos hallazgos podrían sugerirnos la relativa seguridad del uso del FEN intratecal con respecto a la DR.

Encontramos una incidencia de desaturación alta y significativa con la administración de hipnóticos en dos de los cuatro pacientes que presentaron el evento, lo que corresponde al $50 \%$. En nuestro estudio la prioridad de los procedimientos fue cirugía programada, lo cual no permite establecer diferencias concluyentes entre la posibilidad de EA y el tipo de cirugía (programada o urgencia), que tampoco se describe en la literatura. El prurito presentado como segundo EA en frecuencia, $(6,7 \%)$ de preferencia en salas de cirugía, autolimitado, sin necesidad de intervención y las dosis de FEN no fueron diferentes al resto de pacientes. Ya la incidencia de prurito como EA común fue descrito en múltiples series en trabajo de parto y cirugía urológica por Wells y col. ${ }^{25}$ y Khan y col. ${ }^{26}$ respectivamente.

Las náuseas como EA más común en nuestro estudio, tampoco tuvo relación con la dosis. No hubo aumento de la frecuencia según el bloqueo sensitivo anestésico ni por tabaquismo. La relación con el nivel anestésico elevado fue de tres episodios de náuseas $(11,1 \%)$ del total de 27 presentados y dos habían recibido hipnóticos $(7,4 \%)$.

El vómito ocurrió en 2,2\% de los pacientes, sin relación con bloqueo sensitivo alto y las dosis fueron iguales al resto de pacientes. En dos del total de siete (28\%) fueron asociados con administración de hipnóticos. Hamber ${ }^{23}$ menciona en su revisión un posible efecto protector contra el vómito con el uso de opioides intratecales. Techanivate $^{4}$ y col. y Jain y col. ${ }^{27}$ en sus estudios comparan las mezclas de BUP con FEN vs BUP con solución salina, hallando menor frecuencia de vómito en los grupos que usaron FEN, lo que soporta el planteamiento de Hamber. En nuestro estudio no tuvimos grupo control por lo cual no podemos dar validez a este hallazgo.

La presencia de sonda vesical se relacionó con el tipo de cirugía más que con retención urinaria. Uno solo la requirió en recuperación, secundaria a retención.

La bradicardia se presentó en siete casos $(2,2 \%)$, cuatro en salas de cirugía y tres en recuperación. Del total, sólo uno tuvo un nivel anestésico alto que puede tener relación con el bloqueo de fibras cardioaceleradoras, pero no existió cambio importante en las dosis de FEN intratecal o el uso de hipnóticos. El mayor grupo de población con EA perteneció a las clasificaciones ASA I y II que obedece al patrón de distribución natural que acude a cirugía programada. 
Conviene anotar que la bradicardia ocasional requirió administración de atropina con respuesta satisfactoria y si bien no puede afirmarse en este estudio una asociación franca con el uso de FEN, debe tenerse en cuenta como ya otros lo habían valorado dentro de sus estudios, asumiendo medidas farmacológicas para corregirla sin desenlaces fatales, como se lee en Kusneimi y col. ${ }^{1}$ Por otra parte, la administración de opioides intratecales puede expresar otros EA como escalofrío, evaluado dentro de las variables en estudios como del de Techanivate y col. ${ }^{4}$ y que no fue contemplado en nuestro trabajo.

\section{Conclusiones}

La frecuencia de DR que encontramos en nuestro estudio está en el mismo rango que la reportada en la literatura; sin embargo hay que considerar que no existe consenso en la manera como se mide esta variable, además de encontrar una importante asociación entre el uso de hipnóticos y el total del evento reportado. Otros EA se hallaron con menor frecuencia que la reportada. La mayoría ocurrieron en el grupo que pertenece al ASA I y II, que son los pacientes que con más frecuencia acuden a cirugía programada y a su vez conforman el mayor porcentaje de pacientes de nuestro estudio. Se debe siempre tener presente la posibilidad de bradicardia en el enfermo con bloqueo neuroaxial.

\section{Referencias}

1. Kuusniemi KS, Pihlajamaki KK, Pitkanen MT, Helenius HY, Kirvela OA The use of bupivacaine and fentanyl for spinal anesthesia for urologic surgery. Anesth Analg. 2000 Dec; 91(6):1452-6.

2. Gwirtz KH, Young JV, Byers RS, Alley C, Levin K, Walker SG, et al. The safety and efficacy of intrathecal opioid analgesia for acute postoperative pain seven years' experience with 5969 surgical patients at Indiana University Hospital. Anesth Analg. 1999 Mar; 88(3):599-604

3. Herpolsheimer A, Schretenthaler J. The use of intrapartum intrathecal narcotic analgesia in a community-based hospital. Obstet Gynecol. 1994 Dec; 84(6):931-6.

4. Techanivate A, Urusopone $\mathrm{P}$, Kiatgungwanglia $\mathrm{P}$, Kosawiboonpol R Intrathecal fentanyl in spinal anesthesia for appendectomy. J Med Assoc Tha 2004 May;87(5):525-30.

5. Miller R, editor. Miller's anesthesia. 7a ed. Philadelphia : Elsevier Churchill Livingstone, 2009

6. Brown, David L. Spinal, Epidural, and Caudal anesthesia. In: Miller R, editor. Miller's anesthesia. 7a ed. Philadelphia : Elsevier Churchill Livingstone, 2009

7. Keats AS. The ASA classification of physical status - a recapitulation Anesthesiology. 1978 Oct; 49(4): 233-6.

8. Grundy SM, Cleeman JI, Merz CN, Brewer HB, Jr., Clark LT, Hunninghake DB, et al. Implications of recent clinical trials for the National Cholesterol
Education Program Adult Treatment Panel III guidelines. Circulation. 2004 Jul 13; 110(2): 227-39.

9. Obesity, Sleep Apnea, the Airway and Anesthesia. In: Hagber CA. Benumof's airway management. 2nd ed. Philadelphia : Mosby; 2007.

10. American Heart Association. ECC Committee, Subcommittees and Task Forces. 2005 American Heart Association Guidelines for Cardiopulmonary Resuscitation and Emergency Cardiovascular Care. Circulation. 2005 Dec 13;112(24 Suppl):IV1-203.

11. Reuben SS, Dunn SM, Duprat KM, O'Sullivan P. An intrathecal fentanyl dose-response study in lower extremity revascularization procedures. Anesthesiology. 1994 Dec;81(6):1371-5

12. Vaughan DJ, Ahmad N, Lillywhite NK, Lewis N, Thomas D, Robinson PN. Choice of opioid for initiation of combined spinal epidural analgesia in labour—fentanyl or diamorphine. Br J Anaesth. 2001 Apr;86(4):567-9

13. Urwin SC, Parker MJ, Griffiths R. General versus regional anaesthesia for hip fracture surgery: a meta-analysis of randomized trials. Br J Anaesth. 2000 Apr; 84(4):450-5

14. Ben-David B, Frankel R, Arzumonov T, Marchevsky Y, Volpin G. Minidose bupivacaine-fentanyl spinal anesthesia for surgical repair of hip fracture in the aged. Anesthesiology. 2000 Jan; 92(1):6-10.

15. Fernandez-Galinski D, Rue M, Moral V, Castells C, Puig MM. Spinal anesthesia with bupivacaine and fentanyl in geriatric patients. Anesth Analg. $1996 \mathrm{Sep} ; 83(3): 537-41$

16. Fournier R, Van GE, Weber A, Gamulin Z. A comparison of intrathecal analgesia with fentanyl or sufentanil after total hip replacement. Anesth Analg. 2000 Apr;90(4):918-22.

17. Gupta A, Axelsson K, Thorn SE, Matthiessen P, Larsson LG, Holmstrom B, et al. Low-dose bupivacaine plus fentanyl for spinal anesthesia during ambulatory inguinal herniorrhaphy: a comparison between $6 \mathrm{mg}$ and $7.5 \mathrm{mg}$ of bupivacaine. Acta Anaesthesiol Scand. 2003 Jan;47(1):13-9.

18. Ginosar Y, Mirikatani E, Drover DR, Cohen SE, Riley ET. ED50 and ED95 of intrathecal hyperbaric bupivacaine coadministered with opioids for cesarean delivery. Anesthesiology 2004 Mar; 100(3):676-82

19. Korhonen AM, Valanne JV, Jokela RM, Ravaska P, Korttila K. Intrathecal hyperbaric bupivacaine $3 \mathrm{mg}$ + fentanyl 10 microg for outpatient knee arthroscopy with tourniquet. Acta Anaesthesiol Scand. 2003 Mar;47(3):342-6

20. Stamenkovic D, Geric V, Djordjevic M, Raskovic J, Slavkovic Z, Randjelovic $\mathrm{T}$, et al. Subarachnoid morphine, bupivacaine and fentanyl as part of combined spinal-epidural analgesia for low anterior resection. A prospective, randomised, double-blind clinical trial. Anaesth Intensive Care. 2009 Jul;37(4):552-60.

21. Sudarshan G, Browne BL, Matthews JN, Conacher ID. Intrathecal fentanyl for post-thoracotomy pain. Br J Anaesth. $1995 \mathrm{Jul} ; 75(1): 19-22$

22. Ummenhofer WC, Arends RH, Shen DD, Bernards CM. Comparative spinal distribution and clearance kinetics of intrathecally administered morphine, fentanyl, alfentanil, and sufentanil. Anesthesiology. 2000 Mar;92(3):739-53.

23. Hamber EA, Viscomi CM. Intrathecal lipophilic opioids as adjuncts to surgical spinal anesthesia. Reg Anesth Pain Med. 1999 May;24(3):255-63.

24. Bernards CM. Recent insights into the pharmacokinetics of spinal opioids and the relevance to opioid selection. Curr Opin Anaesthesiol. 2004 Oct; 17(5):441-7

25. Wells J, Paech MJ, Evans SF. Intrathecal fentanyl-induced pruritus during labour: the effect of prophylactic ondansetron. Int J Obstet Anesth. 2004 Jan;13(1):35-9.

26. Khan FA, Hamdani GA. Comparison of intrathecal fentanyl and buprenorphine in urological surgery. J Pak Med Assoc. 2006 Jun;56(6):277-81

27. Jain K, Grover VK, Mahajan R, Batra YK. Effect of varying doses of fentanyl with low dose spinal bupivacaine for caesarean delivery in patients with pregnancy-induced hypertension. Int J Obstet Anesth. 2004 Oct; $13(4): 215-20$ 